\title{
Influence of perfusate temperature on nasal potential difference
}

\author{
Inez Bronsveld ${ }^{1,10}$, François Vermeulen ${ }^{2,10}$, Dorotha Sands ${ }^{3}$, Teresinha Leal ${ }^{4}$, \\ Anissa Leonard ${ }^{5}$, Paola Melotti ${ }^{6}, Y^{2}$ asmin Yaakov ${ }^{7}$, Roel de Nooijer ${ }^{1}, K^{2}$ ris De \\ Boeck $^{2}$, Isabelle Sermet ${ }^{8}$, Michael Wilschanski ${ }^{7}$ and Peter G. Middleton ${ }^{9}$ on \\ behalf of the European Cystic Fibrosis Society - Diagnostic Network \\ Working Group
}

Affiliations: 'University Medical Centre Utrecht, Utrecht, The Netherlands, ${ }^{2} \mathrm{CF}$ Centre, University Hospital Gasthuisberg, Leuven, ${ }^{4}$ Dept of Clinical Chemistry, Université Catholique de Louvain, Brussels, and ${ }^{5}$ Pediatric Pulmonology \& Cystic Fibrosis Unit, Université Catholique de Louvain, Brussels, Belgium. ${ }^{3}$ Institute of Mother and Child, Dept of Pediatrics, Warsaw, Poland. ${ }^{6}$ Cystic Fibrosis Centre, Azienda Ospedaliera Universitaria Integrata, Verona, Italy. ${ }^{7}$ Hadassah University Hospital, Jerusalem, Israel. ${ }^{8}$ CF Centre, Hôpital Necker, Paris, France. ${ }^{9}$ Westmead Millennium Institute, University of Sydney, Westmead, Australia. ${ }^{10}$ Both authors contributed equally.

Correspondence: F. Vermeulen, Dept of Paediatrics, University Hospital Gasthuisberg, Catholic University of Leuven, Herestraat 49, 3000 Leuven, Belgium. E-mail: francois.vermeulenduzleuven.be

ABSTRACT Nasal potential difference (NPD) quantifies abnormal ion transport in cystic fibrosis. It has gained acceptance as an outcome measure for the investigation of new therapies.

To quantify the effect of solution temperature on NPD, we first examined the effect of switching from room temperature $\left(20-25^{\circ} \mathrm{C}\right)$ to warmed $\left(32-37^{\circ} \mathrm{C}\right)$ solutions and vice versa during each perfusion step. Secondly, standard protocols were repeated at both temperatures in the same subjects.

Changing solution temperature did not alter NPD during perfusion with Ringer's solution $(<1 \mathrm{mV})$ $(\mathrm{p}>0.1)$. During perfusion with zero chloride solution, changing from room temperature to warmed solutions tended to decrease absolute NPD (i.e. it became less negative) by $0.9 \mathrm{mV}$ ( $\mathrm{p}>0.1$ ); changing from warmed to room temperature increased NPD by $2.1 \mathrm{mV}(\mathrm{p}<0.05)$. During isoprenaline perfusion, changing from room temperature to warmed solutions increased NPD by $1.5 \mathrm{mV}(\mathrm{p}<0.01)$ and from warmed to room temperature decreased NPD by $1.4 \mathrm{mV}(\mathrm{p}<0.05)$.

For full protocols at room temperature or warmed in the same subjects, mean values were similar $(n=24)$. During warmed perfusion, group results for total chloride response had a larger standard deviation. As this increased variability will probably decrease the power of trials, this study suggests that solutions at room temperature should be recommended for the measurement of NPD.

@ERSpublications

This study recommends that perfusate solutions for measuring nasal potential difference should be at room temperature http://ow.ly/kMKzR 


\section{Introduction}

Measurement of nasal potential difference (NPD) evaluates ion transport across human airway epithelium in vivo. NPD measurement has gained acceptance as a diagnostic tool for cystic fibrosis (CF) $[1,2]$ and has been used to measure the effects of disease modifying treatments $[3,4]$ on transepithelial ion transport.

Over the past 20 years, many groups have measured NPD using the technique initially described by KNOWLES et al. [5], with modifications to the site of measurement and the exact details of the exploring electrode, perfusate temperature and perfusate composition. Over the past 10 years, standard operating procedures have been developed in the USA $[6,7]$.

Warming of the perfusates, as originally described by KNOwLES et al. [5] but not by others [8], is part of the Cystic Fibrosis Foundation standard operating procedure. A previous study compared the NPD protocol performed in different subject groups studied with warmed and room temperature perfusates. This study showed a slightly smaller response to zero chloride solution and a larger response to isoprenaline when the solutions were warmed, but there was large intersubject variability [9].

During the development of the standard operating procedure for NPD measurements by the European Cystic Fibrosis Society Diagnostic Network (ECFS-DN) Working Group, the large variability in this study was noted, so a second study was undertaken to examine the effect of perfusate temperature on the NPD. Six centres of the ECFS-DN Working Group participated. The perfusate temperature was changed during each step of the NPD protocol, using a similar design to a recent study comparing different chloride concentrations in the perfusate [10]. In a separate series of studies, a standard protocol was performed with either warmed or room temperature solutions, in the same subjects on different days. The overall aim of this study was to determine the influence of using warmed compared with room temperature solutions on NPD.

\section{Methods}

Subjects

Six ECFS-DN sites recruited a total of 69 healthy subjects, mean age 32 years (range 16-60 years). All were nonsmokers and were studied $\geqslant 4$ weeks after any upper respiratory tract infection.

NPD was measured according to the methods of KNOwLEs et al. [11] $(n=57)$, using an exploring electrode below the inferior turbinate with a subcutaneous agar-filled reference electrode, or the London method [8] $(n=12)$, measuring along the floor of the nose compared with a surface reference electrode. The standardised protocol for NPD comprises sequential perfusion of 1) physiological salt solution to measure baseline NPD, followed by the responses to 2) $100 \mu \mathrm{M}$ amiloride, 3) zero chloride solution with $100 \mu \mathrm{M}$ amiloride and 4) zero chloride solution with $100 \mu \mathrm{M}$ amiloride and $10 \mu \mathrm{M}$ isoprenaline. The total chloride response is the cumulative change in NPD during steps 3 and 4 . To warm the solutions, the perfusion line was placed in a thermostatically controlled water bath or in a warmed water sleeve using counter-current flow to achieve the designated perfusate temperature. Initial validation studies showed that the temperature of the solutions at the tip of the catheter during perfusion was $20-25^{\circ} \mathrm{C}$ for room temperature and $32-37^{\circ} \mathrm{C}$ for warmed solutions. Following the change of temperature between room temperature and warmed the temperature at the tip changed by $12-14^{\circ} \mathrm{C}$ in both directions within $30-40 \mathrm{~s}$.

\section{Protocols}

To directly compare the response to temperature changes during a particular step of the perfusion protocol, solutions were perfused at one of the two temperatures for $\geqslant 4 \mathrm{~min}$, changed to the other temperature for $4 \mathrm{~min}$, and then back to the initial temperature for another $4 \mathrm{~min}$. The opposite temperature sequence was applied in the other nostril, in random order.

In the second series of experiments, the full perfusion protocol was measured in the same subjects, with either room temperature or warmed solutions, on different days, in random order.

\section{Statistical analysis}

To simplify discussion, the NPD is reported as absolute values of mean $\pm S D$, with the lumen always negative. Comparison between the different temperatures was made using paired t-tests. In parallel protocols, the results for each individual were averaged to give a single change for temperature increase or decrease for that individual subject.

All studies were conducted with approval from the local hospital ethics committees, and informed consent was obtained from each participant. 
TABLE 1 Change in nasal potential difference (NPD) values after temperature changes from room temperature (RT) to warmed (W) solutions and from warmed to room temperature solutions during perfusion with Ringer's, zero chloride and isoprenaline solutions

\begin{tabular}{lccc} 
& Subjects n & \multicolumn{2}{c}{ Change in NPD $\mathbf{m V}$} \\
\cline { 2 - 3 } & & RT to $\mathbf{~}$ & W to RT \\
\hline Ringer & 37 & $-0.1 \pm 1.5$ & $-0.2 \pm 1.6$ \\
Zero chloride & 25 & $-0.9 \pm 2.9$ & $2.1 \pm 4.4^{*}$ \\
Isoprenaline & 44 & $1.5 \pm 3.2^{* *}$ & $-1.4 \pm 3.7^{*}$ \\
\hline
\end{tabular}

Data are presented as mean $\pm S D$, unless otherwise stated. $p$-values are given for paired t-tests. ${ }^{*}: p<0.05$; $* *: p<0.01$.

\section{Results}

Temperature changes during perfusion

The effect of temperature changes during the perfusion are given in table 1 . No significant effect of temperature changes was seen during perfusion with baseline Ringer's solution for either the room temperature-warmed-room temperature sequence: room temperature $12.6 \pm 6.0 \mathrm{mV}$, warmed $11.9 \pm 5.8 \mathrm{mV}$ and room temperature $11.8 \pm 5.8 \mathrm{mV}$ or the warmed-room temperature-warmed sequence: warmed $13.1 \pm 7.5 \mathrm{mV}$, room temperature $12.7 \pm 7.0 \mathrm{mV}$ and warmed $13.1 \pm 8.1 \mathrm{mV}$.

However, during perfusion with zero chloride solution, warming the perfusate (change from room temperature to warmed) decreased the absolute NPD with a mean decrease in NPD of $0.9 \mathrm{mV}$. Cooling the perfusate (change from warmed to room temperature) increased the NPD by $2.1 \mathrm{mV}$. For the individual sequences, the absolute responses were: room temperature-warmed-room temperature sequence: room temperature $24.3 \pm 14.3 \mathrm{mV}$, warmed $24.5 \pm 14.3 \mathrm{mV}$ and room temperature $26.0 \pm 15.4 \mathrm{mV}$; or warmedroom temperature-warmed sequence: warmed $22.2 \pm 12.3 \mathrm{mV}$, room temperature $25.0 \pm 11.8 \mathrm{mV}$ and warmed $23.0 \pm 11.7 \mathrm{mV}$.

Conversely, warming the isoprenaline perfusate increased NPD and cooling the perfusate decreased absolute NPD: room temperature-warmed-room temperature sequence: room temperature $36.4 \pm 13.5 \mathrm{mV}$, warmed $38.3 \pm 14.8 \mathrm{mV}$ and room temperature $37.7 \pm 12.6 \mathrm{mV}$; or warmed-room temperature-warmed sequence warmed: $35.7 \pm 16.0 \mathrm{mV}$, room temperature $33.6 \pm 14.5 \mathrm{mV}$ and warmed $34.7 \pm 14.7 \mathrm{mV}$.

The mean \pm SD changes in NPD during temperature changes are reported in table 1 . Time courses of the NPD after changes between room temperature and warmed solutions and back to room temperature during isoprenaline perfusion are shown in figure 1 .
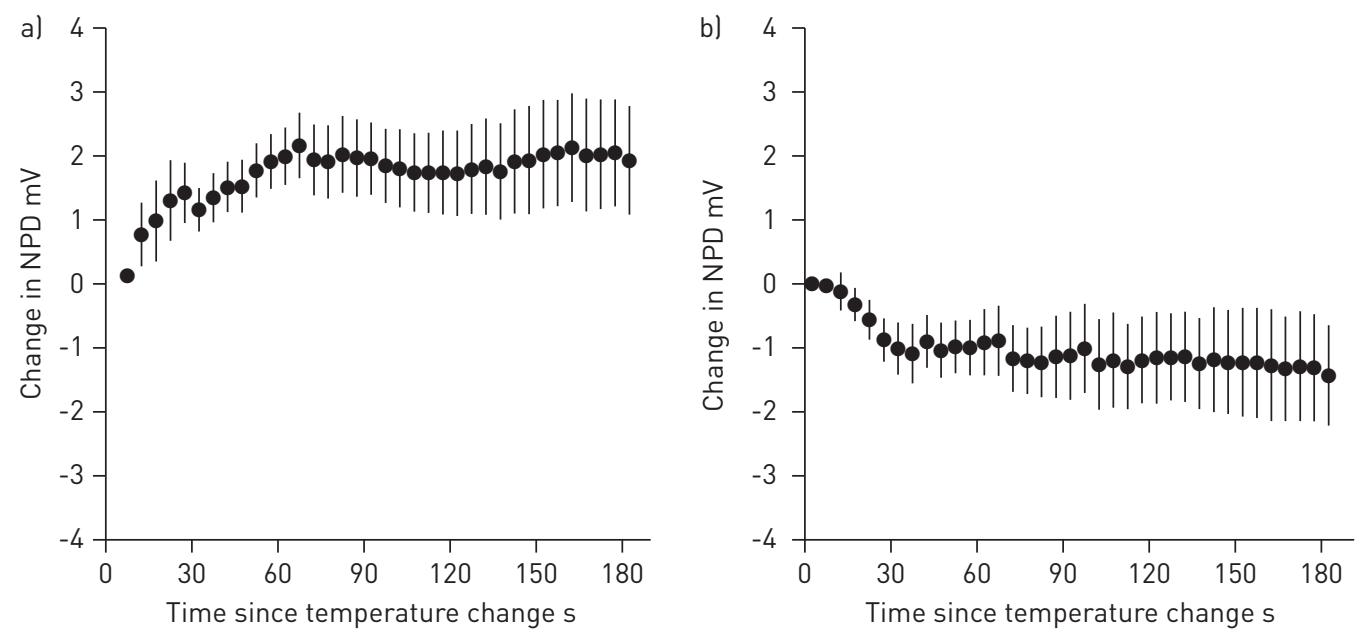

FIGURE 1 Time course of the mean \pm SEM relative change in potential after changing the temperature of solutions during perfusion with isoprenaline a) from room temperature to warmed and b) from warmed to room temperature. $n=16$ patients from one site. 
TABLE 2 Group means of nasal potential difference (NPD) parameters and mean differences measured during perfusion with room temperature (RT) and warmed (W) solutions

Subjects $n$

NPD mV

Difference $\mathrm{mV}$

$95 \% \mathrm{Cl}$ for mean

RT W

\begin{tabular}{lccccc}
\hline Baseline potential & & & & & $-1.3 \pm 4.9$ \\
Amiloride response & 24 & $12.8 \pm 7.5$ & $11.5 \pm 5.8$ & $0.1 \pm 3.5$ & $-3.3-0.8$ \\
Zero chloride response & 24 & $-7.2 \pm 4.4$ & $-7.1 \pm 4.1$ & $-1.5 \pm 18.2$ & $-1.3-1.6$ \\
Isoprenaline response & 21 & $25.0 \pm 13.6$ & $23.6 \pm 15.9$ & $3.6 \pm 11.7$ & $-1.7-8.9$ \\
Total chloride response & 21 & $9.8 \pm 8.4$ & $13.4 \pm 12.4$ & $-1.1 \pm 16.8$ & $-8.2-6.0$ \\
\hline
\end{tabular}

Data are presented as mean $\pm \mathrm{SD}$, unless otherwise stated. All $p$-values for paired t-tests are not significant.

Complete perfusion protocol with room temperature and warmed solutions in the same subjects In 24 subjects, NPD measurement was obtained with room temperature and warmed solutions on different days in random order, as shown in table 2. Individual differences between NPD values are plotted in figure 2, showing the large intra-subject differences between measurements.

\section{Discussion}

This study has confirmed the small but measurable difference in chloride responsiveness when the NPD is measured using warmed solutions. Similar to the previous report by BoYLE et al. [9], NPD during perfusion with low-chloride solution was smaller (less negative) when using $37^{\circ} \mathrm{C}$ solutions, while NPD during perfusion with warmed isoprenaline solution was higher. Finally, testing the same subjects on different days with warmed or room temperature solutions showed differences of -15-20 mV in total chloride response, probably reflecting day-to-day variability rather than the different temperatures tested. The differences observed are in line with previous studies of day-to-day variability $[2,12]$.

The important new finding in the current study is that changing perfusate temperature during the measurement allowed delineation of a small response without the difficulty of day-to-day and subject-tosubject variability. This parallels the recent demonstration that low-chloride responses with $0 \mathrm{mM}$ versus $6 \mathrm{mM} \mathrm{Cl}^{-}$solutions gave an extra $2 \mathrm{mV}$ response [10].

This multicentre study has shown that baseline NPD is not altered by the temperature of the perfusate. Similar to the changes shown previously [9], the potential difference during perfusion of warmed solutions was slightly smaller (less negative) during perfusion with zero chloride, and slightly larger (more negative) during perfusion with isoprenaline. There was a $1.1 \mathrm{mV}$ difference in the total chloride response, which results in a $\sim 3 \%$ greater response at $37^{\circ} \mathrm{C}$. Yet this increased response was at the expense of increased between-subject variability, with the group standard deviation increasing by $4 \mathrm{mV}$.

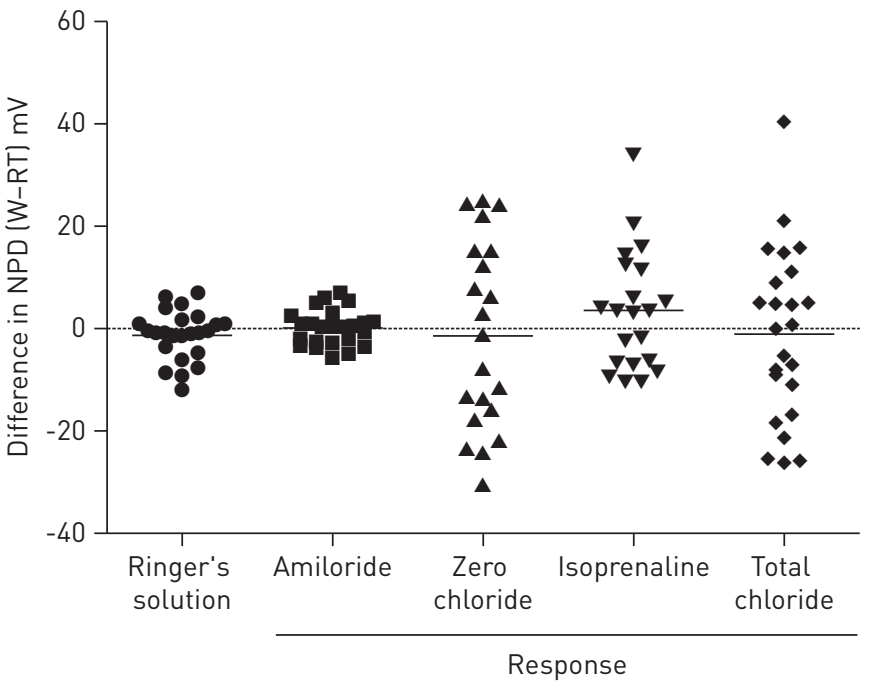

FIGURE 2 Individual differences between nasal potential difference (NPD) parameters measured with room temperature (RT) and warmed (W) solutions in the same subjects. Horizontal bars depict mean difference. 
As the total chloride responses at $37^{\circ} \mathrm{C}$ were larger than those at $20^{\circ} \mathrm{C}$, it could be argued that NPD perfusates should be warmed, as warmed solutions provide the largest difference between $\mathrm{CF}$ and non-CF subjects. However, this needs to be balanced against the increased between-subject variability seen during the $37^{\circ} \mathrm{C}$ perfusion. This increased the variability of the responses and may thus affect the statistical power of studies of new treatments for CF. As the power of a study to determine a change is dependent on the ratio between the size of a response and the variability of that response, higher variability may well outweigh any advantage of a larger response.

The use of $37^{\circ} \mathrm{C}$ solutions for the NPD had its origins in measurement from Ussing chambers and other in vitro testing equipment, where changing apical and basolateral temperatures changes ion transport. However, the situation is different for the NPD where the basolateral surface is always perfused at $37^{\circ} \mathrm{C}$ in vivo. Given that one of the main functions of the nose is to warm the inspired air, intranasal surface temperatures are kept around $30-32^{\circ} \mathrm{C}$ when breathing air at room temperature [13]. In two of our subjects, intranasal temperature was measured at $31-32^{\circ} \mathrm{C}$ during perfusion with solutions at room temperature, showing that the effect of solution temperature on the intranasal temperature is small.

The main aim of a standard operating procedure is to harmonise a technique to simplify the steps involved, to reduce the risk of error in the procedure and thus ensure that all investigators are performing the same testing across different sites in different countries. This allows replication during multicentre studies, with maximal accuracy and minimal variability. In turn this gives the smallest number of subjects to be recruited to conclusively demonstrate a response, with the least chance of Type I or Type II errors. Thus, the aim of a standard operating procedure is to have the simplest, easiest system which generates the largest response with least variability.

In conclusion, this study has demonstrated that, in volunteer subjects expressing a functional CF transmembrane conductance regulator, warmed solutions result in slightly larger total chloride responses, but at the expense of a larger variability. We conclude that the use of warmed solutions has little net overall advantage and thus room temperature solutions should be recommended for simplicity, stability of recording, and reproducibility across centres in different parts of Europe.

\section{References}

1 De Boeck K, Derichs N, Fajac I, et al. New clinical diagnostic procedures for cystic fibrosis in Europe. J Cyst Fibros 2011; 10: Suppl. 2, S53-S66.

2 Middleton PG, House HH. Measurement of airway ion transport assists the diagnosis of cystic fibrosis. Pediatr Pulmonol 2010; 45: 789-795.

3 Accurso FJ, Rowe SM, Clancy JP, et al. Effect of VX-770 in persons with cystic fibrosis and the G551D-CFTR mutation. N Engl J Med 2010; 363: 1991-2003.

4 Kerem E, Hirawat S, Armoni S, et al. Effectiveness of PTC124 treatment of cystic fibrosis caused by nonsense mutations: a prospective phase II trial. Lancet 2008; 372: 719-727.

5 Knowles M, Gatzy J, Boucher R. Increased bioelectric potential difference across respiratory epithelia in cystic fibrosis. N Engl J Med 1981; 305: 1489-1495.

6 Rowe SM, Clancy JP, Wilschanski M. Nasal potential difference measurements to assess CFTR ion channel activity. Methods Mol Biol 2011; 741: 69-86.

7 Standaert TA, Boitano L, Emerson J, et al. Standardized procedure for measurement of nasal potential difference: an outcome measure in multicenter cystic fibrosis clinical trials. Pediatr Pulmonol 2004; 37: 385-392.

8 Middleton PG, Geddes DM, Alton EW. Protocols for in vivo measurement of the ion transport defects in cystic fibrosis nasal epithelium. Eur Respir J 1994; 7: 2050-2056.

9 Boyle MP, Diener-West M, Milgram L, et al. A multicenter study of the effect of solution temperature on nasal potential difference measurements. Chest 2003; 124: 482-489.

10 House HH, Middleton PG. Impact of different chloride and glucose solutions on nasal potential difference. Pediatr Pulmonol 2009; 44: 645-648.

11 Knowles MR, Paradiso AM, Boucher RC. In vivo nasal potential difference: techniques and protocols for assessing efficacy of gene transfer in cystic fibrosis. Hum Gene Ther 1995; 6: 445-455.

12 Vermeulen F, Proesmans M, Feyaerts N, et al. Nasal potential measurements on the nasal floor and under the inferior turbinate: does it matter? Pediatr Pulmonol 2011; 46: 145-152.

13 Liener K, Leiacker R, Lindemann J, et al. Nasal mucosal temperature after exposure to cold, dry air and hot, humid air. Acta Otolaryngol 2003; 123: 851-856. 